\title{
Cirurgias de feminização e de masculinização facial: repercussões forenses
}

\author{
Feminization and masculinization facial surgeries: forensic repercussions \\ Cirugías de feminización y de masculinización facial: repercusiones forenses
}

Recebido: 21/09/2021 | Revisado: 30/10/2021 | Aceito: 06/10/2021 | Publicado: 10/10/2021

Franciéllen de Barros

ORCID: https://orcid.org/0000-0001-5460-4334 Universidade Estadual Paulista "Júlio de Mesquita Filho", Brasil

E-mail: fran.dbrs@gmail.com

Mônica da Costa Serra

ORCID: https://orcid.org/0000-0001-8820-2982 Universidade Estadual Paulista "Júlio de Mesquita Filho", Brasil

E-mail: monica.serra@unesp.br

Barbara Kuhnen

ORCID: https://orcid.org/0000-0003-2586-4772 Universidade Estadual Paulista "Júlio de Mesquita Filho", Brasil E-mail: kuhnenbarbara@gmail.com

Clemente Maia da Silva Fernandes

ORCID: https://orcid.org/0000-0002-5401-6265 Universidade Estadual Paulista “Júlio de Mesquita Filho", Brasil E-mail: c.face@terra.com.br

\begin{abstract}
Resumo
Indivíduos que se caracterizam por identificação forte e persistente com o sexo oposto (disforia de gênero) frequentemente procuram cirurgiões para se submeterem a cirurgia de redesignação sexual e/ou para acentuar características faciais específicas de gênero, modificando cirurgicamente as características da face, na busca de serem aceitos na sociedade como parte do sexo antagônico. Os procedimentos cirúrgicos que têm como objetivo modificar e reconstruir as características ósseas faciais, tal como dos tecidos moles, estão sendo realizados para tornar os rostos de mulheres trans mais femininos (cirurgia de feminização facial) e de homens trans mais masculinos (masculinização facial). Estes procedimentos podem trazer consequências forenses, devido a dificuldades na estimativa do sexo, no que diz respeito à necessidade de reconhecimento do indivíduo por meio de Reconstrução Facial Forense, e consequentemente, em identificar pessoas submetidas a esse tipo de cirurgia. Tais consequências, embora importantes, são pouco consideradas em estudos científicos. Com isso, este estudo traz uma abordagem teórica e reflexiva sobre os aspectos e considerações forenses no reconhecimento e identificação humana, de indivíduos transexuais submetidos a cirurgias de feminização e de masculinização facial.
\end{abstract}

Palavras-chave: Antropologia forense; Odontologia legal; Identificação de vítimas; Feminização e masculinização; Pessoas transgênero.

\begin{abstract}
Individuals who are characterized by strong and persistent identification with the opposite sex (gender dysphoria) often seek plastic surgeons for sex reassignment and/or to accentuate gender-specific facial features, surgically modifying facial features, wishing to be accepted in society as part of antagonistic sex. Surgical procedures aimed at modifying and reconstructing facial bone characteristics, as well as soft tissues, are being performed to make the face of trans women more feminine (facial feminization surgery) and trans men more masculine (facial masculinization). These procedures may have forensic consequences, due to difficulties in estimating the sex, with regard to the possible need for recognition of the individual through Forensic Facial Reconstruction, and consequently, in identifying people who have undergone this type of surgery. Such consequences, although important, are little considered in scientific studies. Thus, this study brings a theoretical and reflective approach to forensic aspects and considerations in human recognition and identification of transsexual individuals who underwent feminization to masculinization facial surgeries.

Keywords: Forensic anthropology; Forensic dentistry; Identification of victims; Feminization and masculinization; Transgender persons.

\section{Resumen}

Las personas que se caracterizan por una identificación fuerte y persistente con el sexo opuesto (disforia de género) a menudo buscan cirujanos para someterse a una cirugía de la reasignación de sexo y / o para acentuar rasgos faciales específicos de género, modificando quirúrgicamente los rasgos del rostro, deseando ser aceptados en la sociedad como parte del sexo antagónico. Se están realizando procedimientos quirúrgicos destinados a modificar y reconstruir las características óseas faciales, así como los tejidos blandos, para hacer que los rostros de las mujeres trans sean más
\end{abstract}


femeninos (cirugía de feminización facial) y de los hombres trans más masculinos (masculinización facial). Estos procedimientos pueden tener consecuencias forenses, debido a las dificultades en la estimación del sexo, en cuanto a la necesidad de reconocimiento del individuo a través de la Reconstrucción Facial Forense, y en consecuencia, identificar a las personas sometidas a este tipo de cirugía. Tales consecuencias, aunque importantes, son poco consideradas en los estudios científicos. Así, este estudio aporta un acercamiento teórico y reflexivo a los aspectos y consideraciones forenses en el reconocimiento e identificación humanos de las personas transexuales sometidas a cirugías de feminización y masculinización facial.

Palabras clave: Antropología forense; Odontología forense; Identificación de víctimas; Feminización y masculinización; Personas transgénero.

\section{Introdução}

A face humana apresenta grande variabilidade, resultante da evolução , o que permite que cada indivíduo seja único e facilmente reconhecível (Sanders, 2014). Torna-se um dos meios mais importantes e naturais que transmitem informações individuais. Assim, todos os dias a face é utilizada como recurso de reconhecimento de pessoas (Biometric Technology Today, 2003). Por influência disto, distinguir indivíduos tornou-se mais fácil, pois a evolução beneficiou a diversidade facial devido à grande variedade existente. A face possui um aspecto central no instinto social do ser humano (Lents, 2014).

Formada por um conjunto de tecidos, como ossos, cartilagens, músculos e pele, entre outros, a face apresenta ainda textura e cor variadas, sendo essas diferenças que fazem com que cada indivíduo se torne único, diferente dos demais. Consequentemente, forma-se um conjunto de informações que auxiliará no reconhecimento das pessoas (Verzé, 2009). Além do conjunto de características que formam a face humana, esta deriva de variações genéticas específicas que compões características como, tamanho do nariz e largura do rosto (Shaffer et al., 2016). Segundo Kim et al. (2013), a face contribui para a identidade de cada indivíduo e para o autoconhecimento, pois é um dos elementos primordiais do corpo humano na obtenção de informações individuais. Logo, é possível diferenciar uma pessoa da outra devido também às suas características faciais. Para McNeill (1998), a face representa muitos aspectos evidentes e subconscientes de fisionomia, exibindo processos biológicos e psicológicos, como também idade, ancestralidade e sexo.

Desde a antiguidade até os dias atuais, constata-se acentuada preocupação da humanidade com a estética facial e a beleza, o que leva a um crescente número de buscas por procedimentos e intervenções cirúrgicas. Nesta perspectiva, a história da cirurgia para pessoas transgênero é razoavelmente longa no contexto dos procedimentos cirúrgicos modernos, por exemplo, a cirurgia genital (cirurgia de redesignação sexual) para pacientes transgênero (Deschamps-Braly, 2018). A proporção de indivíduos que se identifica como transgênero ou com diversidade de gênero tem aumentado nos últimos anos, sendo crescente a demanda por procedimentos estéticos - desde os minimamente invasivos e até a transformação facial (Ginsberg, 2017; Lai et al., 2020). Durante o processo de transição, indivíduos transgênero podem buscar modificações estéticas faciais, na busca de uma aparência correspondente ao seu gênero (Ginsberg et al., 2016; Dhingra et al., 2019), pois desejam que sua aparência facial transpareça como se sentem a respeito de si mesmos (Ginsberg et al., 2016). Segundo Morrison et al. (2016), a procura por cirurgia de feminização facial (CFF) aumentou em prevalência, e a cirurgia de masculinização facial (CMF) possui menor demanda.

A CFF é realizada por meio de vários procedimentos estabelecidos na cirurgia estética para feminilizar o crânio e os tecidos moles da face. Já a CMF tem sido aplicada mais recentemente, a homens trans para masculinizar a face e a cartilagem tireóide (Deschamps-Braly, 2018).

À medida em que aumenta o número de indivíduos submetidos a tais procedimentos, amplia também o número de casos forenses que podem requerer estimar, através dos ossos, o sexo das pessoas que se submeteram a tais intervenções. Por isso a importância das pesquisas antropológicas sobre a modificação da estrutura óssea. A ausência de dados pode dificultar a identificação, e até levar a estimativas forenses equivocadas, levando a malefícios sociais, jurídicos e patrimoniais (Kuhnen et al., 2020). 
A porção óssea craniana que dá base à face não é uma estrutura neutra sobre a qual são colocados tecidos moles sexualmente diferenciados; ao contrário, é o próprio crânio que fornece a diferenciação sexual facial através da sua arquitetura (Becking et al., 2007). A CFF altera os tecidos moles e duros (ossos) faciais e, visto que a face, especialmente sua estrutura óssea, é um elemento importante de identificação (incluindo o dimorfismo sexual), as mudanças cirúrgicas podem ter consequências forenses significativas (Kuhnen et al., 2020), o mesmo ocorrendo com a CMF.

No campo forense, identificação é o termo usado para determinar as características ou identidade de um objeto ou indivíduo. Portanto, os processos de identificação estão diretamente relacionados à obtenção de características ou qualidades para caracterizar a individualidade que distingue um determinado objeto de interesse de outros da mesma natureza (Frari, 2008). Uma das principais respostas que os especialistas devem esclarecer ao realizar as análises com vistas à identificação de um corpo é o sexo do indivíduo. Este é um dos primeiros procedimentos que devem ser realizados no âmbito pericial para o processo de identificação, pois irá orientar corretamente o percurso da investigação na busca da identidade do indivíduo (Zilio et al., 2013).

Assim, o conjunto de procedimentos cirúrgicos interessa aos especialistas durante as análises forenses, devido às alterações na estrutura craniana. Quando a análise é realizada em mulheres transgênero que foram submetidas a procedimentos cirúrgicos faciais, a estrutura facial fornecerá características femininas, mas a morfologia pélvica revelará o sexo biológico com características masculinas (Buchanan, 2014), e o mesmo ocorre em homens trans. Isto faz com que seja necessário o conhecimento de tais modificações feitas pelos processos cirúrgicos, assim como as marcas resultantes de tais intervenções, permitindo a comprovação de incoerências entre a morfologia pélvica e craniana (Buchanan, 2014).

Se for encontrado um corpo ou ossada e existirem suspeitas sobre a identidade do falecido, será feita a comparação dos dados ante mortem com os dados post mortem, na tentativa de estabelecer sua identidade (Fernandes, 2010). Mas, por outro lado, quando inexistirem suspeitas de quem seja o indivíduo, um dos métodos realizados no âmbito forense é a Reconstrução Facial Forense (RFF), que constrói as características faciais a partir de um crânio seco não identificado, com o objetivo de possibilitar o reconhecimento do falecido, cujo sexo, ancestralidade e idade no momento da morte precisam ser anteriormente estimados. Feito o reconhecimento, realiza-se o método de identificação (Fernandes, 2010; Wilkinson, 2010; Fernandes et al., 2012; Kim et al., 2013). À vista disso, durante a análise pericial o perito deve se atentar às características das estruturas ósseas cranianas, buscando indícios (como cicatrizes ósseas) de que aquele indivíduo foi submetido a procedimento cirúrgico facial - esta informação auxiliará na confecção da RFF e no processo de identificação.

O presente estudo traz uma abordagem teórica e reflexiva sobre os aspectos e considerações forenses no reconhecimento e identificação humana de indivíduos transexuais submetidos a CFF e CMF.

\section{Metodologia}

Este trabalho baseou-se em uma revisão descritiva da literatura com abordagem qualitativa (Pereira et al., 2018), constituindo um extenso estudo e permitindo a inclusão de pesquisas experimentais e não experimentais e de literatura teórica e empírica, para aprofundar o conhecimento sobre a temática estudada. A busca abrangente foi realizada nas bases de dados primárias PubMed, SciELO, LILACS e Web of Science. Os descritores utilizados foram "'cirurgia de feminização facial", "cirurgia de masculinização facial", "identificação humana", "reconhecimento", "cirurgia de redesignação de gênero", "crânio" e "reconstrução facial forense".

Inicialmente, os descritores foram pesquisados individualmente e, em seguida, foram realizados cruzamentos entre eles. A amostra selecionada incluiu artigos, dissertações e teses que estivessem disponíveis na íntegra, publicados em português ou inglês, em qualquer período, e referentes ao tema estudado. Os critérios de exclusão foram artigos publicados em idiomas diferentes e não relacionados ao tema estudado. A pesquisa foi classificada e avaliada, finalizando com a interpretação dos resultados e síntese do conhecimento. 


\section{Sexo e Gênero}

Sexo e gênero são identificadores independentes um do outro, e não estão necessariamente correlacionados. O sexo é determinado pelas características biológicas do indivíduo, e pode ser classificado como masculino, feminino ou intersexo (Byers, 2017; Killermann, 2014; Shapiro, 1981; Statistics Canada, 2020). Sendo assim, é biologicamente quantificável, por meio da análise de órgãos, hormônios e cromossomos. Um homem é uma pessoa com cromossomos e testículos XY, uma mulher é uma pessoa com cromossomos XX, ovários e útero, e intersexo é uma pessoa com qualquer combinação de tais características biológicas (Killermann, 2014; Newman \& Cross, 2018).

O gênero é influenciado pelos aspectos sociais, comportamentais, psicológicos e culturais da vida (Newman \& Cross, 2018; Shapiro, 1981; Statistics Canada, 2020). Culturalmente, o gênero inclui o que a sociedade considera "feminino" ou "masculino", bem como comportamentos e aparências apropriados relacionados aos termos "homem” e "mulher" (Moore, 2003; Schall et al., 2020). Estes comportamentos são construções sociais, e consequentemente não podem ser considerados naturais ou predeterminados, mas comportamentos que mudam à medida em que cada pessoa cresce e aprende em seu ambiente (Moore, 2003).

\section{Dimorfismo Sexual do Crânio}

Quando são comparados crânios masculinos e femininos, verifica-se que as dimensões cranianas dos homens são maiores que das mulheres, sendo a face masculina mais quadrada, angulada e musculosa na vista frontal. A fronte geralmente exibe protuberância frontal significativa, com fortes cristas supraorbitais, e a linha da mandíbula tem um ângulo proeminente com laminação do osso devido às inserções do músculo masseter, além de ser mais larga. O ângulo formado na glabela entre a área frontal da testa e o nariz é frequentemente agudo. As sobrancelhas são retas e tendem a sentar-se no nível da borda orbital superior. As bochechas masculinas são achatadas e o queixo frequentemente mais longo, quadrado e angulado. A cartilagem tireóidea é mais proeminente no homem e no entalhe forma um ângulo de $90^{\circ}$ (Altman, 2012; Telang, 2020).

Já a face feminina é mais arredondada ou oval, com formas suaves e curvas. O mento é pequeno e pontudo, além de apresentar a fronte lisa com o ângulo formado na glabela entre a área frontal da testa. As sobrancelhas são arqueadas, principalmente na região do terço lateral, e ficam bem acima da borda orbital superior. As bochechas são bastante proeminentes, sendo mais anteriores e mais altas. O nariz é menor e mais curto com ângulo nasolabial mais obtuso (Altman, 2012; Telang, 2020).

À vista disso, para Fisher et al. (2020), a face masculina tem características faciais maiores e mais angulares, com a fronte mais acentuada e maior protuberância nas sobrancelhas; e na face feminina ao contrário, características menores, estreitas e suaves. Na região frontal, os procedimentos envolvem recuo da parede do seio frontal, avanço do couro cabeludo e elevação da sobrancelha (Capitán et al., 2014; Ousterhout, 1987; Becking et al., 2007). Para Habal (1990), em relação as órbitas, no sexo masculino são menores e menos arredondadas em comparação ao sexo feminino. A porção nasal é maior nos homens, assim como o formato da face mais largo e o queixo mais quadrado. O sexo feminino apresenta um nariz com ângulo glabelar mais obtuso e uma ponta mais projetada, e o formato facial inferior e o queixo mais estreitos e triangulares. A cartilagem tireóidea é mais proeminente no sexo masculino (Becking et al., 2007; Capitán et al., 2014).

Considerando tais diferenças, para feminizar a face é necessária a redução óssea de certas áreas do crânio, como a saliência frontal, cristas supraorbitais, mento e ângulo da mandíbula, bem como acentuar a gordura nos tecidos moles, para corresponder e atingir a extensão feminina "ideal" de acordo com a perspectiva do paciente (Becking et al., 1996; Plemons, 2012; Capitán et al., 2014). Para Telang (2020), para feminizar a face é necessária a correção de protuberância frontal, rinoplastia, contorno de mandíbula e mento e raspagem da proeminência laríngea da cartilagem tireoidea (popularmente conhecida como pomo-de-adão). Segundo Altman (2012), é necessário a redução da testa, avanço do couro cabeludo, implantes de bochecha, 
ressecção do corpo adiposo da bochecha, elevação da sobrancelha, elevação labial (lifting labial), aumento do enxerto de derme, genioplastia, corte angular e cônico da mandíbula e condrolaringoplastia.

Em contrapartida, para masculinizar a face, os procedimentos cirúrgicos realizados incluem alongamento de fronte, aumento frontal e redução lateral do zigomático, rinoplastia, osteotomia, remodelação da mandíbula ou aumento de mandíbula e realce da cartilagem tireóidea (Kamol Hospital, 2020).

\section{Cirurgias de Feminização e de Masculinização Facial}

O ser humano acentua suas características específicas de gênero através de maquiagem, penteados, joias e até mesmo com procedimentos cosméticos, para melhorar e intensificar a sua aparência. Assim, indivíduos que se caracterizam por identificação forte e persistente com o sexo oposto (disforia de gênero), frequentemente procuram cirurgiões para se submeterem a cirurgia de redesignação sexual e/ou para acentuar características faciais específicas de gênero, modificando cirurgicamente as características das suas faces, e serem classificados corretamente (Fisher et al., 2020).

A cirurgia reconstrutiva genital e as intervenções endócrinas foram os primeiros procedimentos destinados a mudar o gênero de uma pessoa, pois evidenciam as genitais com a questão da masculinidade ou feminilidade. Mas, no entanto, em 1980, uma nova abordagem de procedimentos foi elaborada para modificar uma área do corpo que tem um papel central na avaliação e atribuição do sexo na vida cotidiana: a face (Plemons, 2014).

O procedimento associado à transição transexual, no qual o paciente se submete a uma mudança no corpo para adequar o sexo anatômico ao psicológico, é denominado de cirurgia de redesignação de gênero (CRG) conhecida também como cirurgia de redesignação sexual (CRS) (Plemons, 2012). É realizado um conjunto de procedimentos por meio dos quais a aparência física e as características sexuais primárias são modificadas cirurgicamente para se parecerem as do sexo oposto (Ainsworth \& Spiegel, 2010). Como resultado disso, o desejo de se submeter a CRS tem sido frequentemente considerado como atribuição de indivíduos transgênero (Becking et al., 1996) e, à medida em que os procedimentos se tornaram mais prevalentes, houve um aumento nas buscas por informações relacionadas aos mesmos.

Porém, existem outras características corporais que podem interferir na aceitação social bem-sucedida como membro do sexo oposto como, por exemplo, as características faciais (Becking et al. 1996), que são consideradas de extrema importância para aceitação em público, apesar de parecerem, para alguns autores, menos importantes do que a reatribuição da genitália (Shams \& Motamedi, 2009).

Segundo Defreyne et al. (2017), por mais que a CRS tenha revelado redução na disforia de gênero, nos níveis de depressão e ansiedade e nas tentativas de suicídio nos indivíduos submetidos a tal procedimento, quando tais números são comparados à média nacional, permanecem dez vezes maior (Haas et al., 2014). Isso pode ser explicado pelo fato de que por mais que haja uma diminuição na disforia de um indivíduo que se submeteu a cirurgia de redesignação sexual, a censura social perdura. Diante disso, a CFF tem também a função de equiparar as características corporais com sua identidade, diminuindo a disforia de gênero, facilitando a transição e o reconhecimento social (Facque et al., 2019), assim como a CMF.

Entretanto, torna-se nulo o impacto da redesignação sexual em termos de mudanças na forma como a sociedade reconhece e responde ao gênero corporificado da pessoa na vida social cotidiana, pois os órgãos genitais permanecem ocultos. Sendo assim, se o paciente deseja, através de intervenções médico-cirúrgicas, ser reconhecido pelo gênero desejado, a mudança mais significativa e visível pela sociedade está voltada para alterações cirúrgicas na face (Plemons, 2012), tais como a CFF e a CMF.

A CFF é definida como uma combinação de cirurgias ósseas e de tecidos moles da face que tem como objetivo modificar as principais diferenças referentes ao dimorfismo sexual - características faciais masculinas e femininas. Essa linha de intervenção fornece um equilíbrio estético para a face masculina, tornando-a mais feminina, sendo comumente realizada em 
pacientes que foram diagnosticados por identificação forte e persistente com o gênero oposto e/ou os que possuem transtorno de identidade de gênero, comumente conhecido como não-conformidade de gênero, com a finalidade de melhorar a autoestima do paciente e contribuindo significativamente no desenvolvimento da sua qualidade de vida na sociedade (Ousterhout, 1987; Tugnet et al., 2007; Spiegel, 2008; Ainsworth \& Spiegel, 2010; Coleman et al., 2012; Capitán et al., 2014). Da mesma forma, porém menos frequentemente, a cirurgia de CFF também pode ser realizada em mulheres cisgênero que desejam apenas feminizar certos aspectos faciais (Telang, 2020).

Em 1980, o cirurgião craniomaxilofacial Ousterhout desenvolveu pela primeira vez os procedimentos usados na CFF, após uma paciente que realizou a CRS e que, embora tivesse mudado seu sexo genitalmente, não observou impacto algum em como as pessoas percebiam seu gênero na vida social cotidiana, questionando se era possível transformar seu rosto de aparência masculina com características femininas. Diante de tal questionamento, Ousterhout examinou textos de antropologia física, estudos longitudinais de crescimento craniofacial e uma coleção de crânios secos, com o objetivo de desenvolver um conjunto de medidas e proporções que entendeu serem necessários para definir as diferenças cranianas entre homens e mulheres. Como resultado disso, uma série de técnicas foram desenvolvidas, que lhe permitiriam reconstruir pacientes. Quando Ousterhout começou a realizar a CFF, seguiu o modelo aceito pela medicina transexual em meados do século XX, entendendo que feminizar a face de homens era um procedimento para auxiliar mulheres transgênero (Plemons, 2014; Plemons, 2017).

Comparando ambas as cirurgias, a reconstrução genital é entendida como o procedimento para mudar o sexo de uma mulher trans, e a feminização facial simplesmente contribui na melhora da sua aparência, ao torná-la mais congruentemente feminina. Sendo assim, Ousterhout, com o passar dos anos, compreendeu a eficácia da CFF de forma diferente pois, para suas pacientes, tornar-se mulher não dependia da anatomia e fisiologia da genitália feminina, e sim, de ser reconhecida como mulher pela sociedade (Plemons, 2014; Plemons, 2017).

Neste sentido, para Plemons (2012), o que diferencia a CFF dos demais procedimentos destinados a feminilizar a face é a modificação e reconstrução dos ossos faciais, bem como dos tecidos moles. Porém, a parte óssea fornece a estrutura subjacente da face, e é onde se situa a feminilidade sendo, portanto, o crânio que diferencia a arquitetura do sexo facial. Em geral não é possível alcançar a feminização desejada modificando apenas tecidos moles faciais (embora as alterações dos mesmos seja uma parte essencial da CFF), mas é necessário alterar as características masculinas ósseas subjacentes (Plemons, 2012).

Por consequência, a CFF traz benefícios para os indivíduos que se submetem ao procedimento, e tem se mostrado como um campo crescente no atendimento de pacientes trans em todo o mundo, trazendo melhora na experiência psicológica e integração social dos mesmos (Morrison et al., 2016). Vários estudos demonstraram alta satisfação de pacientes com tal intervenção (Ousterhout, 1987; Becking et al., 2007; Shams \& Motamedi, 2009; Li et al., 2012; Capitán et al., 2014; Balaji, 2016; Fisher et al., 2020).

Após serem submetidos a CFF, os pacientes esperam ter suas faces com características mais femininas, para serem reconhecidos por outras pessoas como mulheres e serem inseridos neste gênero. Certos de que os procedimentos terão poder transformador no reconhecimento cotidiano, em muitos casos, diversos pacientes optam pelas mudanças faciais antes,ou mesmo em substituição à cirurgia genital reconstrutiva, pois alterar a aparência facial simboliza uma transição muito mais profunda do que a transformação pessoal e íntima (Plemons, 2017).

No estudo de Shams e Motamedi (2009), dez pacientes com idades entre 20 e 32 anos foram submetidos à CFF usando diretrizes cirúrgicas básicas. Durante o procedimento, os pacientes passaram por várias alterações de tecidos moles e duros, de acordo com a avaliação pré-operatória e parâmetros padrão, obtendo resultados satisfatórios na aparência. Da mesma forma, Fisher et al. (2020) em seu estudo mostraram que após a CFF, os pacientes eram propícios a serem identificados como sendo do gênero feminino e apresentavam mais confiança, sendo classificados de acordo com o gênero corretamente, quase com a mesma frequência de pacientes cisgênero, pertencentes ao grupo controle. 
Recentemente houve um aumento na prevalência de mulheres transgênero que procuram cirurgiões para realizar mudanças nas características faciais através da CFF. Neste caso, estudos recentes mostram que tais pacientes desejam realizar este procedimento na mesma proporção em comparação com a cirurgia de afirmação genital (Morrison et al., 2016). Porém, pouco se sabe sobre a prevalência da realização de CFF (Canner et al., 2018), pois é difícil categorizar o número de cirurgias. Em consideração a isso, Teixeira et al. (2020) realizaram um estudo com o intuito de buscar, pela primeira vez, uma análise dos dados do Google Trends em relação ao interesse global na CFF. Os autores observaram que houve um aumento geral no interesse entre os procedimentos para feminizar a face, bem como na cirurgia de redesignação sexual.

Para Ousterhout \& Deschamps-Braly (2019), por mais que ultimamente a porcentagem de mulheres trans que recorrem aos procedimentos da CFF não esteja clara, devido ao aumento na procura de tal cirurgia, são necessários o treinamento e a educação aprimorada dos cirurgiões. Para se obter sucesso na cirurgia, é necessário que o especialista tenha capacidade e habilidades precisas em cirurgia craniomaxilofacial e de tecidos moles, compreendidos aqui o conhecimento da complexidade da anatomia loco-regional e sua destreza cirúrgica, e compatibilizando-os com a compreensão da expectativa do paciente, além da percepção pública do resultado do procedimento cirúrgico (Balaji, 2016; Telang, 2020; Fisher et al., 2020).

Sob outra perspectiva, o uso de terapia hormonal por si só pode não ser suficiente para fornecer características tradicionalmente masculinas à face feminina. Todavia, a CMF em homens trans é opção ideal para atingir maior grau de masculinização. É composta por um conjunto de procedimentos cirúrgicos estéticos e reconstrutivos, e tem como objetivo remodelar as características faciais femininas, para atingir uma aparência com características masculinas, que não podem ser alcançadas apenas com a terapia hormonal, considerando as proporções faciais e a anatomia geral do paciente (The international center - for transgender care; Sayegh et al., 2019; American Society Of Plastic Surgeons, 2021). Os pacientes que se submetem a tal procedimento normalmente melhoram a autoestima e a autoimagem, juntamente com uma capacidade aprimorada de serem aceitos na sociedade como homens (The international center - for transgender care; Sayegh et al., 2019).

Desse modo, Sayegh et al. (2019) buscaram na literatura e resumiram as técnicas operatórias empregadas em CMF, com objetivo de fornecer uma abordagem algorítmica para o tratamento desses pacientes. A busca das técnicas cirúrgicas e resultados da CMF em pacientes transgênero e cisgênero, foi feita nos bancos de dados PubMed, EMBASE e Medline, com publicação até julho de 2018. Também foi feita a coleta sobre os dados demográficos dos pacientes, acompanhamento, técnicas operatórias, complicações e resultados. Dos vinte e quatro estudos encontrados, apenas quinze preencheram os critérios de inclusão. Dentre estes quinze, dois abordavam os resultados de 7 sujeitos (6 homens trans e 1 homem cis) que se submeteram a procedimentos para masculinizar as características faciais - os indivíduos estavam geralmente satisfeitos e não houve complicações. Os demais estudos revisaram as técnicas cirúrgicas utilizadas na população cisgênero. Segundo os autores, os procedimentos atuais para masculinizar a face em pacientes cisgênero podem ser considerados nos pacientes transgênero, e bons resultados têm sido alcançados. No entanto, são necessárias mais pesquisas sobre técnicas e medidas de resultados objetivos de procedimentos de masculinização facial na população trans.

Ousterhout (2011) realizou 6 procedimentos de masculinização facial através de masculinização da fronte, do mento e do ângulo mandibular, em homens cisgênero para fins cosméticos, e publicou suas técnicas em 2011. A única diferença encontrada entre os 6 pacientes é que em 2 foi usada a técnica de enxerto ósseo para aumentar o ângulo mandibular, enquanto nos demais foram empregados implantes para aumento do ângulo. Em todos os pacientes foi usado metacrilato de metila para aumentar a fronte. A masculinização do mento foi realizada por meio de genioplastia. Todas as cirurgias foram consideradas satisfatórias pelos pacientes.

Da mesma forma, Deschamps-Braly et al. (2017), ao realizarem uma revisão da literatura, não identificaram nenhum relato prévio de homens trans submetidos à CMF. Estes autores realizaram, em 2017, a primeira CMF em um paciente transgênero homem. Foi realizado um procedimento para aumentar a cartilagem tireóidea, usando cartilagem de costela autóloga 
para masculinizar mais as características de cabeça e pescoço, pois a adição do "pomo-de-adão" realça a aparência masculina facial de uma pessoa biologicamente do sexo feminino. Além disso, foram invertidos os métodos normalmente usados para feminilizar as características faciais masculinas, modificando a fronte, o nariz e o mento para masculinizar as características faciais através dos métodos realizados em homens cisgênero. Os autores concluíram que houve satisfação do paciente com a CMF e com aumento da cartilagem tireóidea, realizado em uma segunda etapa.

Quando é feita a comparação da prevalência dos dois procedimentos, o número de indivíduos submetidos CFF excede o quantitativo de submetidos à CMF, fato este que se reflete na literatura, que apresenta mais informações sobre as técnicas de feminização facial (Ousterhout, 1987; Tugnet et al. 2007; Spiegel, 2008; Ainsworth \& Spiegel, 2010; Coleman et al. 2012; Capitán et al. 2014; Plemons, 2014; Plemons, 2017; Teixeira et al., 2020; Telang, 2020). Diante disso, Facque et al. (2019) realizaram um estudo com o objetivo de discutir as diferenças anatômicas, bem como as intervenções cirúrgicas e não cirúrgicas atuais utilizadas para masculinizar ou feminizar a face. Os autores destacaram a importância de tais cirurgias de confirmação de gênero facial e procedimentos auxiliares para que seja promovida a transição e integração social, bem como para alinhar a anatomia de uma pessoa com sua identidade. Além disso, ressaltaram a variedade de procedimentos cirúrgicos e não cirúrgicos que podem masculinizar ou feminizar a anatomia facial de um indivíduo.

Da mesma forma, Deschamps-Braly (2019) descreveu os procedimentos necessários para feminizar ou masculinizar um rosto. Para este autor, a CFF e a CMF apresentam-se como ferramentas poderosas para mudar as características sexuais da face. Não obstante, os procedimentos são complexos, sendo um desafio para o cirurgião responsável em criar uma aparência que corresponda à percepção interna do indivíduo de si mesmo, porém, são procedimentos satisfatórios para os indivíduos.

\section{Identificação Humana e Reconstrução Facial Forense}

Nas Ciências Forenses, o processo de estabelecer a identidade dos indivíduos requer a utilização de métodos científicos baseados na somatoscopia (variações qualitativas) e somatometria (variações quantitativas) do corpo humano (İşcan, 2005; França, 2011). O conjunto de caracteres físicos, funcionais e psíquicos (natos ou adquiridos) que individualiza uma pessoa, tornando-a diferente das demais e idêntica a si própria, é designado identidade. Entretanto, o processo que determina a identidade e permite distinguir uma pessoa de todas as outras é denominado de identificação (Champod \& Meuwly, 2000; Kanchan \& Krishan, 2013). No campo pericial, a identificação pessoal é indispensável tanto por razões legais como humanitárias, e pode ser iniciada antes de se determinar a causa da morte (Carvalho et al., 2009). Desta forma, a identificação permite chegar à identidade humana através do uso de técnicas e meios propícios (Holobinko, 2012).

Existem alguns métodos de identificação que visam a determinar a identidade humana. Tais métodos permitem distinguir uma pessoa de todas as outras, pois cada indivíduo tem características únicas. Os métodos de identificação são comparativos - para executá-los é necessário haver um primeiro registro (obtido antes da morte) e a seguir, uma comparação com o segundo registro (obtido após a morte) (Fernandes, 2010). Existem vários métodos que podem ser empregados para identificar cadáveres; no entanto, os considerados primários são a datiloscopia, a Odontologia Legal e a análise de DNA (Interpol, 2018).

A Odontologia Legal compreende a aplicação da ciência odontológica para identificar restos humanos desconhecidos, ao comparar dados anteriores com dados posteriores à morte. Este é o principal método de identificação de vítimas em desastres de grande escala (Prajapati et al., 2018). Rugoscopia palatina e queiloscopia são algumas das técnicas utilizadas; emprego de exames imaginológicos (como radiografias e tomografias computadorizadas) para avaliação de seios paranasais e estágios de mineralização dentária, entre outros, são importantes exemplos das possíveis atuações desta especialidade (Verrísimo et al., 2021; Barros et al., 2021a; Kuhnen et al., 2021; Gioster-Ramos et al., 2021; Barros et al., 2021b; Barros et al., 2021c; Santos et al., 2021; Neves et al., 2021). 
Quando um corpo é encontrado e levado ao Instituto Médico-Legal (IML) sem condições de ser reconhecido visualmente, devido ao estado em que foi encontrado (como casos de carbonizados, esqueletizados e putrefeitos) a aplicação de conhecimentos antropológicos é importante para, por exemplo, traçar o perfil biológico (que inclui informações como idade, ancestralidade, estatura e sexo), eventualmente excluindo suspeitos e auxiliando as investigações (İşcan, 2005; Fernandes, 2010; Silva, 2019). Uma das etapas fundamentais no trabalho da Antropologia Forense é a estimativa do sexo para a obtenção do perfil biológico, realizada por meio de análises em componentes ósseos como a pelve e o crânio., que fornecem relevantes informações sobre o dimorfismo sexual (Geller, 2005; Hu et al., 2006; Saini et al., 2011; Luo et al., 2013), sendo a análise realizada por meio de inspeção visual/morfológica (métodos não métricos ou qualitativos) ou pela análise métrica (métodos métricos ou quantitativos) (Kemkes \& Göbel, 2006). É necessário destacar que, quando os profissionais avaliam as características sexuais, não é o gênero que estão considerando, e sim o sexo biológico (Schall et al., 2020).

A RFF é uma técnica projetada para reconstruir manualmente ou digitalmente a face de um sujeito, a partir de crânios secos não identificados. Seu principal objetivo é levar ao reconhecimento de cadáveres que precisam ter sua identidade estabelecida (Fernandes, 2010; Fernandes et al., 2013; Jones, 2014; Haresh et al., 2015; Herrera et al., 2017). Desse modo, quando o indivíduo não foi previamente identificado pelos métodos tradicionais, esta técnica pode ser o último recurso utilizado pelos profissionais (Evison et al., 1998; Fernandes et al., 2013). Assim, estabelecido o perfil biológico, a realização de Reconstrução Facial Forense (RFF) em muito poderá contribuir com a busca da identidade do indivíduo, pois esta técnica permitirá que a sua face reconstruída seja distribuída ao público através da mídia, buscando o seu reconhecimento e, consequentemente, aumentando as chances de identificação (Fernandes, 2010; Fernandes et al., 2013).

Porém, deve-se compreender que a técnica de RFF não leva à identificação do cadáver, mas proporciona o reconhecimento do indivíduo, que poderá ser identificado em seguida, por métodos de identificação (Bulut et al., 2014; Drgáčová et al., 2016). Portanto, identificação e reconhecimento são ações distintas. O reconhecimento é a observação direta ou indireta de um corpo feita por familiares ou amigos. Em consequência disso, refere-se a um método subjetivo, pois não é fundamentado em conhecimento técnico-científico, todavia pode ser um método complementar aos processos de identificação. Em contrapartida, a identificação apresenta-se como um método objetivo, visto que leva à identidade do cadáver, a partir de dados científicos, e trabalho comparativo (Santos et al., 2008; Holobinko, 2012).

Entretanto, para realizar a RFF é fundamental o conhecimento das espessuras dos tecidos moles faciais (ETMFs), que recobrem a estrutura óssea do crânio, pois são informações utilizadas como base para reproduzir a face através dos pontos craniométricos (Greef et al., 2006; Domaracki et al., 2006; Codinha, 2009; Cavanagh \& Steyn, 2011; Drgáčová et al., 2016). Assim, há uma variedade de métodos disponíveis para a mensurar as ETMFs, que vão desde a inserção de agulhas ou lâminas em cadáveres, até meios imaginológicos, como radiografia, ultrassom, ressonância magnética (RM) e tomografia computadorizada (TC), sendo que a RM e a TC são as mais precisas (Aulsebrook et al., 1996; El-Mehallawi \& Soliman, 2001; Codinha, 2009; Fourie et al., 2010; Dong et al., 2012; Bulut et al., 2014; Barros et al., 2021c; Kuhnen et al., 2021). As informações sobre essas médias estão fornecidas em tabelas publicadas na literatura científica internacional, possibilitando métodos de reconstrução (Aulsebrook et al., 1995; Wilkinson, 2010). Devido às diferenças significativas entre as espessuras, as tabelas devem ser organizadas de acordo com as variáveis sexo, ancestralidade, idade e estado nutricional (Fernandes, 2010; Fernandes et al., 2013; Kuhnen et al., 2021; Barros et al., 2021). Portanto, tais variáveis devem ser consideradas em estudos que buscam mensurar ETMFs, para que posteriormente essas informações possam ser utilizadas na confecção de RFFs.

Na literatura científica são encontrados estudos que realizaram mensurações e apresentam tabelas de ETMFs para diferentes populações, de indivíduos adultos de ambos os sexos, como por exemplo, norte-americanos brancos e negros (Rhine \& Campbell, 1980; Rhine \& Moore, 1984), para zulus (Aulsebrook et al., 1996), canadenses (Garlie \& Saunders, 1999), egípcios (El-Mehallawi \& Soliman, 2001), australianos (Simpson \& Henneberg, 2002), caucasianos (Greef et al., 2006), indianos (Sahni 
et al., 2008), europeus (Tilotta et al., 2009), japoneses (Utsuno et al., 2010), chineses (Dong et al., 2012), franceses (Guyomarc'h et al., 2013), paquistaneses (Jeelani et al., 2015), tchecos (Drgáčová et al., 2016), alemães (Thiemann et al., 2017) e brasileiros (Barros et al., 2021c), além de outros.

\section{Estimativa do Sexo e Identificação de Indivíduos Trans Submetidos a CFF e CMF}

As alterações faciais e cranianas resultantes de procedimentos de cirurgia de feminização facial podem confundir, prejudicar, retardar ou até mesmo impossibilitar a identificação post mortem (visto que são realizadas modificações ósseas faciais), especialmente quando os tecidos moles não mais estão presentes. A estimativa de sexo é baseada em formas, contornos e medidas ósseas, específicas para os sexos masculino e feminino. Para pessoas transgênero, é necessário examinar minuciosamente o crânio para detectar marcas ou cicatrizes produzidas durante a intervenção cirúrgica. Quando o esqueleto completo é encontrado, outros ossos também são analisados (Kuhnen et al., 2020; Schall et al., 2020). O mesmo acontece em casos de homens trans submetidos a CMF.

Mesmo que a aparência facial, dos tecidos moles de uma pessoa sofra impacto com a terapia hormonal, é o conjunto de procedimentos cirúrgicos que interessa para a estimativa do sexo, devido às alterações na estrutura do esqueleto (Buchanan, 2014). Consequentemente, a análise pericial das mulheres transgênero que passaram por procedimentos de CFF, alterando as características ósseas faciais torna-se um impasse para os peritos, pois o crânio apresentará características femininas, mas a morfologia pélvica transparecerá o sexo biológico com características masculinas (Buchanan, 2014), e o mesmo, de maneira inversa, ocorre em homens transgênero que se submeteram a procedimentos de CMF.

Sendo assim, é importante que os peritos tenham conhecimento das modificações feitas na estrutura do esqueleto, estando atentos aos procedimentos e às marcas deles resultantes. Desta forma, e com essa compreensão, o perito pode considerar a possibilidade de o corpo em análise forense ser de um indivíduo trans (quando existirem incoerências entre a morfologia pélvica e craniana) (Buchanan, 2014).

Neste contexto, Schall et al. (2020) realizaram um estudo com o objetivo de analisar o impacto dos procedimentos da CFF em relação aos métodos métricos de análise craniana com vistas à estimativa do sexo, com o intuito de elaborar diretrizes para identificar corretamente mulheres trans. Os autores observaram que evidências de gênero podem ser detectadas nos esqueletos faciais de indivíduos transgênero que passaram por CFF, e diante disso, os antropólogos forenses devem considerar a possibilidade de o indivíduo em análise ser transgênero, não se limitando a análises e conclusões que se enquadrem apenas no sexo biológico (sexo binário), ao estimarem o sexo de esqueletos de indivíduos não identificados.

Quando uma cirurgia de feminização facial é bem-sucedida, a face de uma mulher trans deve ser indistinguível de uma mulher cisgênero. Em eventual caso de desaparecimento de uma mulher trans, se reportado por alguém que conhece e respeita sua identificação de gênero, haverá o registro de desaparecimento de uma pessoa do sexo feminino. Neste caso, a estimativa do sexo a partir da análise craniana poderá, para peritos mais atentos, condizer com as características da identidade de gênero, o que não ocorrerá com a análise da pelve. (Kuhnen et al., 2020; Schall et al., 2020). O mesmo pode ocorrem em situações análogas com homens trans.

Ainda no caso de homens transgênero, a técnica desenvolvida por Deschamps-Braly et al. (2017) para aumentar a proeminência laríngea da cartilagem tireóidea (conhecida popularmente como pomo-de-adão) usando cartilagem de costela, para dar um perfil e aparência mais masculina, tem sido um grande passo no alcance do gênero facial para homens trans, podendo ser útil na identificação dos mesmos, devido às alterações pós-cirúrgicas que podem aparecer, dependendo dos níveis de desintegração do corpo em análise (Schall et al., 2020). 


\section{Considerações Finais}

Há um aumento na prevalência de indivíduos transgênero que procuram cirurgiões para realizar mudanças nas características faciais por meio de cirurgias de feminização ou masculinização facial. Todavia, pessoas submetidas a tais procedimentos cirúrgicos podem ser vítimas de acidentes fatais ou outras situações podem advir, em que seja necessária a sua identificação a partir de análises ósseas. Assim, diante do número crescente de casos de pessoas trans que se submetem a cirurgias ósseas faciais, para feminilizar ou masculinizar as suas faces, é importante que peritos, especialmente antropólogos forenses, afeitos a trabalhos de estimativa de sexo a partir de elementos ósseos, sobretudo ao perceberem incoerências entre a análise craniana e a análise pélvica, tenham em mente a possibilidade de estarem diante de um caso de indivíduo trans submetido a CFF ou CMF. Ou seja, que não limitem suas análises e conclusões e, ao avaliar corpos não identificados, considerem os indivíduos que não se enquadram nas categorias binárias de sexo.

Em adição a isto, é importante ressaltar que trabalhos de Reconstrução Facial Forense são realizados a partir da estimativa do sexo (e também da idade e ancestralidade). E se, ao traçar o perfil biológico, o perito não se atentar sobre a possibilidade de estar diante de caso de indivíduo transgênero (com a face operada), a RFF realizada posteriormente mostrará a face com a representação do sexo biológico, e não do gênero socialmente assumido, o que pode dificultar, ou mesmo impossibilitar o reconhecimento do indivíduo.

É importante reforçar que a análise do perfil biológico (realizada por meio exame antropológico forense) e a Reconstrução Facial Forense (RFF) não são métodos de identificação. A RFF corresponde a método de reconhecimento. Métodos de identificação (como datiloscopia, análise de dados odontológicos e análise de DNA) são comparativos e, para a consecução dos mesmos, é necessário que haja uma suspeição quanto à identidade do de cujus, para que se possa comparar os seus dados de primeiro registro (ante mortem) com os dados do segundo registro (post mortem). No caso de a estimativa do sexo ter sido equivocada, diminuem as chances de familiares e/ou amigos próximos do de cujus procurarem o serviço pericial que esteja a cargo do caso para relatar que suspeitam de que o corpo (ou restos mortais ou ossada) encontrado possa ser do seu ente querido. Somente a partir da existência de um(a) suspeito(a), métodos de identificação podem ser realizados, e o corpo eventualmente identificado.

Finalmente, vale lembrar que a correta identificação de uma pessoa falecida tem não somente repercussões jurídicas e patrimoniais, mas também sociais e emocionais. E, nesta conjuntura, é importante que o perito tenha consciência da dimensão e repercussão do seu trabalho, auxiliando a Justiça e a sociedade.

\section{Agradecimentos}

O presente trabalho foi realizado com apoio da Coordenação de Aperfeiçoamento de Pessoal de Nível Superior - Brasil (CAPES) - Código de Financiamento 001.

\section{Referências}

Ainsworth, T. A \& Spiegel, J. H. (2010). Quality of life of individuals with and without facial feminization surgery or gender reassignment surgery. Qual Life Res., 19, 1019-24.

Altman, K. (2012). Facial feminization surgery: current state of the art. International Journal of Oral and Maxillofacial Surgery, 41(8), 885-894.

American Society of Plastic Surgeons. (2021). Facial Masculinization Surgery - Female to Male - the goal of facial Masculinization Surgery is to transform the feminine features of the face to a more masculine appearance. https://www.plasticsurgery.org/reconstructive-procedures/facial-masculinization-surgery

Aulsebrook, W. A., Becker, P. J. \& Işcan, M. Y. (1996). Facial soft-tissue thicknesses in the adult male Zulu. Forensic Sci Int., 79 (2), $83-102$.

Aulsebrook, W. A., Işcan, M. Y., Slabbert, J. H. \& Becker, P. (1995). Superimposition and reconstruction in forensic facial identification: a survey. Forensic Sci Int., 75(2-3), 101-20.

Balaji, S. M. (2016). Facial feminization. Ann Maxillofac Surg., 6(2), 158. 
Barros, F., Fernandes, C. M. S., Kuhnen, B., Scarso Filho, J., Gonçalves, M., \& Serra, M. C. (2021)a. Paranasal sinuses and human identification. Research, Society and Development, 10(9), e48710918161.

Barros, F., Serra, M. C., Kuhnen, B., Matos, R. A. \& Fernandes, C. M. S. (2021)b. Orthodontic 2D and 3D frontal sinus imaging records: an important role in human identification. Research, Society and Development, 10(13), e49110313608.

Barros, F., Serra, M. C., Kuhnen, B., Scarso Filho, J., Gonçalves, M., \& Fernandes, C. M. S. (2021)c. Midsagittal and bilateral facial soft tissue thickness: a Cone - Beam Computed Tomography assessment of Brazilian living adults. Forensic Imaging. 200444.

Becking, A. G., Tuinzing, D. B., Hage, J. J. \& Gooren, L. J. (1996). Facial corrections in male to female transsexuals: a preliminary report on 16 patients. J Oral Maxillofac Surg., 54(4), 413-8.

Becking, A. G., Tuinzing, D. B., Hage, J. J. \& Gooren, L. J. (2007). Transgender feminization of the facial skeleton. Clin Plast Surg., 34(3), 557-564.

Buchanan, S. (2014). Bone Modification in Male to Female Transgender Surgeries: Considerations for the Forensic Anthropologist. Dissertação. Faculdade de Agricultura e Mecânica. Louisiana State University.

Bulut, O., Sipahioglu, S. \& Hekimoglu, B. (2014). Facial soft tissue thickness database for craniofacial reconstruction in the Turkish adult population. Forensic Sci Int., 242, 44-61.

Byers, S. N. (2017). Introduction to forensic anthropology (5th ed.). Routledge.

Canner, J. K., Harfouch, O., Kodadek, L. M., Pelaez, D., Coon, D., Offodile, A. C., Haider, A. H. \& Lau, B. D. (2018). Temporal Trends in gender-affirming surgery among transgender patients in the United States. JAMA Surg., 153, 609-616.

Capitán, L., Simon, D., Kaye, K. \& Tenorio, T. (2014). Facial feminization surgery: The forehead. Surgical techniques and analysis of results. Plast Reconstr Surg., 134, 609-19.

Carvalho, S. P. M., Da Silva, R. H. A., Lopes-Júnior, C. \& Peres, A. S. (2009). A utilização de imagens na identificação humana em odontologia legal*. Radiol Bras., 42(2), 125-130.

Cavanagh, D. \& Steyn, M. (2011). Facial reconstruction: soft tissue thickness values for South African black females. Forensic Sci Int., 206(1-3), e1-215.e7.

Champod, C. \& Meuwly, D. The inference of identity in forensic speaker recognition. Speech Communication, 31(2-3), 193-203.

Codinha, S. (2009). Facial soft tissue thicknesses for the Portuguese adult population. Forensic Sci Int., 184(1-3), 80.

Coleman, E., Bockting, W., Botzer, M., Cohen-Kettenis, P. T., DeCuypere, G., Feldman, J., Fraser, L., Green, J., Knudson, G.,Meyer, W. J., Monstrey, S., Adler, R. K., Brown, G. R., Devor, A. H., Ehrbar, R., Ettner, R., Eyler, E., Garofalo, R., Karasic, D. H., Lev A. L., Mayer, G., Meyer-Bahlburg, H., Hall, B. P., Pfaefflin, F., Rachlin, K., Robinson, B., Schechter, L. S., Tangpricha, V., Trotsenburg, M. V., Vitale, A., Winter, S., Whittle, S., Wylie, K. R. \& EK. Zucker, E. K. (2012). Standards of care for the health of transsexual, transgender, and gender-nonconforming people, version 7. Int J Transgend., 13, 165-232.

Defreyne, J., Motmans, J. \& T'Sjoen, G. (2017). Healthcare costs and quality of life outcomes following gender affirming surgery in trans men: a review. Expert Rev Pharmacoecon Outcomes Res ., 17, 543-556.

Deschamps-Braly, J., Sacher, C., Fick, J. \& Ousterhout, D. (2017). First Female-to-Male Facial Confirmation Surgery with Description of a New Procedure for Masculinization of the Thyroid Cartilage (Adam's Apple). Plastic and Reconstructive Surgery, 139(4), 883e-887e.

Deschamps-Braly, J. C. (2018). Facial Gender Confirmation Surgery: Facial Feminization Surgery and Facial Masculinization Surgery. Clin Plast Surg., 45(3), 323-331.

Deschamps-Braly, J. C. (2019). Approach to Feminization Surgery and Facial Masculinization Surgery: Aesthetic Goals and Principles of Management. Journal of Craniofacial Surgery, 30(5), 1352-1358.

Dhingra N, Bonati LM, Wang EB, Chou M, Jagdeo J. (2019)Medical and aesthetic procedural dermatology recommendations for trans gender patients undergoing transition. J Am Acad Dermatol., 80:1712-1721.

Domaracki, M. \& Stephan, C. N. (2006). Facial soft tissue thicknesses in Australian adult cadavers. J Forensic Sci., 51(1), 5-10.

Dong, Y., Huang, L., Feng, Z., Bai, S., Wu, G. \& Zhao, Y. (2012). Influence of sex and body mass index on facial soft tissue thickness measurements of the northern Chinese adult population. Forensic Sci Int., 222(1-3), 396.

Drgáčová, A., Dupej, J. \& Velemínská, J. (2016). Facial soft tissue thicknesses in the present Czech Population. Forensic Sci Int., 260, 106.e1-106.e7.

El-Mehallawi, I. H. \& Soliman, E. M. (2001). Ultrasonic assessment of facial soft tissue thicknesses in adult Egyptians. Forensic Sci. Int., 117(1-2), 99-107.

Evison, M. P., Finegan, O. M. \& Blythe, T. C. (1998). Computerised 3-D facial reconstruction: research update. https://www.researchgate.net/publication/276025876_Computerised_3-D_facial_reconstruction_research_update.

Facque, A. R., Atencio, D. \& Schechter, L. S. (2019). Anatomical Basis and Surgical Techniques Employed in Facial Feminization and Masculinization. Journal of Craniofacial Surgery, 30(5), 1406-1408.

Fernandes, C. M. S. (2010). Análise das reconstruções faciais forenses digitais caracterizadas utilizando padrões de medidas lineares de tecidos moles da facede brasileiros e estrangeiros. Tese. Universidade de São Paulo, São Paulo, Brasil.

Fernandes, C. M. S., Serra, M. C., Silva, J. V. L., Noritomi, P. Y., Pereira, F. D. S. \& Melani, R. F. H. (2012). Tests of one Brazilian facial reconstruction method 
using three soft tissue depth sets and familiar assessors. Forensic Sci Int., 214(1-3), 211e1-7.

Fernandes, C. M. S., Pereira, F. D. S., Silva, J. V. L. \& Serra, M. C. (2013) Is characterizing the digital forensic facial reconstruction with hair necessary? A familiar assessors' analysis. Forensic Sci. Int. 229 (1-3), 164.e1-164.e5.

Fisher, M., Lu, S. M., Chen, K., Zhang, B., Maggio, MD. \& Bradley, J. P. (2020). Facial Feminization Surgery Changes Perception of Patient Gender. Aesthetic Surgery Journal, 40(7), 703-709.

Fourie, Z., Damstra, J., Gerrits, P. O. \& Ren, Y. (2010). Accuracy and reliability of facial soft tissue depth measurements using cone beam computer tomography. Forensic Sci Int., 199(1-3), 9-14.

França, G. V. Medicina Legal. Guanabara Koogan.

Frari, P., Iwashita, A. R., Caldas, J. C. F. G., Scanavin, M. A. \& Júnior, E. D. (2008). A importância do odontolegista no processo de identificação humana de vítima de desastre em massa. Sugestão de protocolo de exame técnico-pericial. Revista Odonto., 16(31), 38-44.

Garlie, T. N. \& Saunders, S. R. (1999). Midline facial tissue thicknesses of subadults from a longitudinal radiographic study. J Forensic Sci., $44(1)$, 61-7.

Geller, P. L. (2005). Skeletal analysis and theoretical complications. World Archaeology, 47(4), 597-609.

Ginsberg, B. A. (2017). Dermatologic care of the transgender patient. Int J Womens Dermatol., 3(1), 65-67.

Gioster-Ramos, M. L., Silva, E. C. A., Nascimento, C. R., Fernandes, C. M. S. \& Serra, M. C. (2021) Técnicas de identificação humana em Odontologia Legal. Research, Society and Development, 10(3), e20310313200.

Greef, S., Claes, P., Vandermeulen, D., Mollemans, W., Suetens, P. \& Willems, G. (2006). Large-scale in-vivo Caucasian facial soft tissue thickness database for craniofacial reconstruction. Forensic Sci Int., 15, 159 Suppl 1, S126-46.

Guyomarc'h, P., Santos, F., Dutailly, B. \& Coqueugniot, H. (2013). Facial soft tissue depths in French adults: variability, specificity and estimation. Forensic Sci Int., 231(1-3), 411.

Haas, A. P., Rodgers, P. L. \& Herman, J. L. (2014). Suicide attempts among transgender and gender non-conforming adults: findings of the national transgender discrimination survey. American Foundation for suicide prevention. 1-18.

Habal, M. B. (1990). Aesthetics of feminizing the male face by craniofacial contouring of the facial bones. Aesthetic Plast Surg., 14(2), 143-150.

Haresh, A. D., Singh, P. \& Song, J. (2015). Soft tissue thickness determination using CBCT in diverse medical disciplines. J Pharm Biomed Sci., 5(12), 96772 .

Herrera, L. M., Strapasson, R. A. P., Zanin, A. A., da Silva, J. V. L. \& Melani, R. F. H. (2017). Comparison among manual facial approximations conducted by two methodological approaches of face prediction. J Forensic Sci., 62(5), 1279-85.

Holobinko, A. (2012). Forensic human identification in the United States and Canada: A review of the law, admissible techniques, and the legal implications of their application in forensic cases. Forensic Science International, 222, 394.e1-394.e13

Hu, K. S., Koh, K. S., Han, S. H., Shin, K. J., Kim, H. J. (2006). Sex Determination Using Nonmetric Characteristics of the Mandible in Koreans. J Forensic Sci., 51(6), 1376-1382.

Interpol. (2018). Disaster Victim Identification Guide. https://www.interpol.int/How-we-work/Forensics/Disaster-Victim-Identification-DVI

İşcan, M. Y. (2005). Forensic anthropology of sex and body size. Forensic Sci Int., 147(2), 107-112.

Jeelani, W., Fida, M. \& Shaikh, A. (2015). Facial Soft Tissue Thickness Among Three Skeletal Classes in Adult Pakistani Subjects. J Forensic Sci., 60(6), 14205.

Jones, G. (2014). Not a yes or no question: critical perspectives on sex and gender in forensic anthropology. Dissertation. University of Windsor, Canada.

Kamol Hospital - Thailand Cosmetic \& Plastic Surgery. Facial Masculinization Surgery (FMS). (2020).

https://www.kamolhospital.com/en/service/female-to-male-surgery/

Kanchan, T. \& Krishan, K. Personal Identification in Forensic Examinations. Anthropol., 2, 114. 2013.

Killermann, S. (2014). Breaking through the binary: Gender explained using continuum. Its Pronounced Metrosexual. https://www.itspronouncedmetrosexual.com/ 2011/11/breaking-through-the-binary-genderexplained-using-continuums/

Kim, Y. J, Park, J. W., Kim, J. M., Park, S. H,, Hwang, J. H., Kim, K. S., Lee, S. Y. \& Shin, J. H. (2013). The Functionality of Facial Appearance and Its Importance to a Korean Population. Arch Plast Surg., 40(6), 715-720.

Kuhnen, B., Barros, F., Fernandes, C. M. S. \& Serra, M. C. (2020). Cirurgia de feminização facial em transexuais: reflexões éticas e forenses. Rev. Bioét., 28(3), 432-9.

Kuhnen, B., Fernandes, C. M. S., Barros, F., Scarso Filho, J., Gonçalves, M., \& Serra, M. C. (2021). Facial soft tissue thickness of Brazilian living subadults. A Cone-Beam Computed Tomography study. Forensic Imaging. 200434.

Lai, Y. C., Kazlouskaya, M. \& Kazlouskaya, V. (2020). Historical and current state of dermatologic care for sexual and gender minority populations. Dermatol Clin., 38(2), 177-183. 
Lents, L. H. The Stunning Diversity of Human Faces. (2014). The Human Evolution Blog. https://thehumanevolutionblog.com/2014/10/01/the-stunningdiversity-of-human-faces/.

Li, J., Hsu, Y., Khadka, U. M. A., Hu, J., Wang, Q. \& Wang, D. (2012). Surgical designs and techniques for mandibular contouring based on categorisation of square face with low gonial angle in orientals. J Plast Reconstr Aesthet Surg., 65(1), e1-e8.

Luo, L., Wang, M., Tian, Y., Duan, F., Wu, Z., Zhou, M., \& Rozenholc, Y. (2013). Automatic sex determination of skulls based on a statistical shape model. Computational and Mathematical Methods in Medicine, 251628.

McNeill, D. (1998). The face: A natural History. New York. Little, Brown.

Moore, H. L. (2003). Understanding sex and gender. In T. Ingold (Eds.), Companion encyclopedia of anthropology: Humanity, culture and social life.Routledge.

Morrison, S.D., Vyas, K.S., Motakef, S., Gast, K.M., Chung, M.T., Rashidi, V., Satterwhite, T., Kuzon, W. \& Cederna, P.S. (2016). Facial feminization: systematic review of the literature. Plast Reconstr Surg., 137, 1759-1770.

Neves, I. S. R., Santiago, A. P. A. C. e. S., Silva, M. I. T., \& Oliveira, E. R. M. (2021). Rugoscopia palatina e seus desafios na identificação humana: Uma revisão integrativa. Research, Society and Development, 10(4).

Newman, T., \& Cross, K. (2018). Sex and gender: What is the difference? Medical News Today. https://www.medicalnewstoday.com/articles/ 232363\#genderdifferences

Ousterhout, D. \& Deschamps-Braly, J. (2019). Special edition of transgender facial surgery. J Craniofac Surg., 30, 1326-1327.

Ousterhout, D. K. (1987). Feminization of the forehead: contour changing to improve female aesthetics. Plast Reconstr Surg., 79(5), 701-713.

Ousterhout, D. K. (2011). Dr. Paul Tessier and facial skeletal masculinization. Ann Plast Surg., 67(6), S10-5.

Pereira, A. S., Shitsuka, Dorlivete Moreira Parreira, F. J. \& Shitsuka, R. (2018). Metodologiada Pesquisa Científica - Licenciatura em Computação. https://repositorio.ufsm.br/bitstream/handle/1/15824/Lic_Computacao_Metodologia-Pesquisa-Cientifica.pdf?sequence=1.

Plemons, E. D. (2012). Making the Gendered Face: The Art and Science of Facial Feminization Surgery. Dissertation. University of California, Beckley.

Plemons, E. D. (2014). Description of sex difference as prescription for sex change: On the origins of facial feminization surgery. Social Studies of Science, $44(5), 657-679$.

Plemons, E. D. (2017). Formations of Femininity: Science and Aesthetics in Facial Feminization Surgery. Medical Anthropology, 36, 7, 629-641.

Prajapati,G., Sarode, S. C., Sarode, G. S., Shelke, P., Awan, K. H. \& Patil, S. (2018). Role of forensic odontology in the identification of victims of major mass disasters across the world: A systematic review. PLOS ONE 13(6): e0199791.

Rhine, J. S. \& Campbell, H. R. (1980). Thickness of facial tissues in American blacks. J Forensic Sci., 25 (4), 847-58.

Rhine, J. S. \& Moore, C. E. (1984) Tables of facial tissues thickness of American Caucasoids in forensic anthropology. Maxwell Museum Tech Ser.

Sahni, D., Sanjeev, Singh, G., Jit, I. \& Singh, P. (2008). Facial soft tissue thickness in northwest Indian adults. Forensic Sci Int., 176(2-3), 137-46.

Saini, V., Srivastava, R., Rai, R. K., Shamal, S. N., Singh, T. B. \& Tripathi, S. K. (2011). Mandibular Ramus: An Indicator for Sex in Fragmentary Mandible. J Forensic Sci., 56(s1), S13-S6.

Sanders, R. (2014). Human faces are so variable because we evolved to look unique. Politics \& Society, Research, Science \& Environment. Berkeley News. https://news.berkeley.edu/2014/09/16/human-faces-are-so-variable-because-we-evolved-to-look-unique/.

Santos, A. N., Falcão, T. N., Lima, L. N. C., Vieira, T. I., \& Santiago, B. M. (2021). A Queiloscopia como técnica de identificação humana: Uma revisão sistemática da literatura. Research, Society and Development, $10(8)$.

Santos, W. D. F., Diniz, P. R. B., Santos, A. C., Martin, C. C. S. \& Guimarães, M. A. (2008). Definições de pontos craniométricos em imagens multiplanares de ressonância magnética (RM) para fins de reconstrução facial forense. Medicina Ribeirão Preto, 41 (1), 17-23.

Sayegh, F. B. A., Ludwig, D. C., Ascha, M., Krishna, V., ShakiR, A., Kwong, J. W., Swanson, M., Evans, M. W., Gatherwright, J. \& Morrison, D. Facial Masculinization Surgery and its Role in the Treatment of Gender Dysphoria. Journal of Craniofacial Surgery, 30(5), 1339-1346.

Schall, J. L., Rogers, T. L., \& Deschamps-Braly, J.C. (2020). Breaking the binary: The identification of trans-women in forensic anthropology. Forensic Science International, 309, 110220 .

Shaffer, J. R., Orlova, E., Lee, M. K., Leslie, E. J., Raffensperger, Z. D., Heike, C. L., Cunningham, M. L., Hecht, J. T., Kau, C. H., Nidey, N. L., Moreno, L. M., Wehby, G. L., Murray, J. C., Laurie, C. A., Laurie, C. C., Cole, J., Ferrara, T., Santorico, S., Klein, O., Mio, W., Feingold, E., Hallgrimsson, B., Spritz, R. A., Marazita, M. L. \& Weinberg, S. M. (2016). Genome-Wide Association Study Reveals Multiple Loci Influencing Normal Human Facial Morphology. PLoS Genet., 12(8), e1006149.

Shams, M. G. \& Motamedi, M. H. K. (2009). Case Report: Feminizing the Male Face. Eplasty., 9, e2.

Shapiro, J. (1981). Anthropology and the study of gender. Soundings: An Interdisciplinary Journal, 64(4), 446-465.

Silva, M. C. (2019). The increasing role of forensic anthropology in the investigation of missing persons, unidentified remains, and cold cases. Thesis. Tulane University, Nova Orleans, Luisiana. 
Research, Society and Development, v. 10, n. 13, e201101320877, 2021

(CC BY 4.0) | ISSN 2525-3409 | DOI: http://dx.doi.org/10.33448/rsd-v10i13.20877

Simpson, E. \& Henneberg, M. (2002). Variation in soft-tissue thicknesses on the human face and their relation to craniometric dimensions. Am J Phys Anthropol., $118(2), 121-33$

Spiegel, J. H. (2008). Challenges in Care of the Transgender Patient Seeking Facial Feminization Surgery. Facial Plastic Surgery Clinics of North America, 16(2), 233-238.

Statistics Canada. (2020). Sex at birth and gender: Technical report on changes for the 2021 census. https://www12.statcan.gc.ca/censusrecensement/2021/ref/98-20- 0002/982000022020002-eng.cfm

Teixeira, J. C., Morrison, S. D., Brandstetter, K. A. \& Nuara, M. J. (2020). Is There an Increasing Interest in Facial Feminization Surgery? A Search Trends Analysis. J Craniofac Surg., 31(3), 606-607.

Telang, P.S. (2020). Facial Feminization Surgery: A Review of 220 Consecutive Patients. Indian J Plast Surg., 53(2), 244-253.

The International Center - for transgender care. Facial Masculinization Surgery FMS. https://thetranscenter.com/transmen/facial-masculinization-surgery-fms/

Thiemann, N., Keil, V. \& Roy, U. (2017). In vivo facial soft tissue depths of a modern adult population from Germany. Int J Legal Med,. 131(5), 1455-1488.

Tilotta, F., Richard, F., Glaunès, J., Berar, M., Gey, S., Verdeille, S., Rozenholc, Y. \& Gaudy, J. F. (2009). Construction and analysis of a head CTscan database for craniofacial reconstruction. Forensic Sci Int., 191(1-3), 112.

Tugnet, N., Goddard, J.C., Vickery, R.M., Khoosal, D. \& Terry, T.R. (2007). Current management of male-to-female gender identity disorder in the UK. Postgrad Med J., 83, 638-42.

Utsuno, H., Kageyama, T., Uchida, K., Yoshino, M., Oohigashi, S., Miyazawa, H. \& Inoue, K. (2010). Pilot study of facial soft tissue thickness differences among three skeletal classes in Japanese females. Forensic Sci Int., 195(1-3), 1-5.

Veríssimo, M. H. G., Rodrigues, M. A., Nóbrega, W. F. S., Barbosa, D. V., Neto, O. M. M., Dias, B. A. S., \& Silva, G. C. B. . (2021). O conhecimento da anatomia versus a Odontologia Legal: Uma revisão integrativa sobre o processo de identificação humana. Research, Society and Development, 10 (7).

Verzé, L. History of facial reconstruction. (2009). Acta Biomed., 80(1), 5-12.

Wilkinson, C. (2010). Reconstrução facial - arte anatômica ou anatomia artística?. Journal of anatomy, 216 (2), 235-250.

Zilio, F., Basualdo, A. \& Cruz, R. A. (2004). Meios de identificação odontolegal. https://www.imed.edu.br/Uploads/66fd6950-4925-442f-a7ba03007be1b860.pdf. 